\title{
Evaluation of Patient Outcomes of High Flow versus Low Flow Nasal Oxygen during Outpatient Bronchoscopy under Conscious Sedation at Thoracic Procedure Suite in Lyell McEwin Hospital
}

\section{Singankutti Mudalige Thanuja Nilushi Priyangika ${ }^{1 *}$, Moayed Alawami ${ }^{2}$ and Shanka Karunarathne ${ }^{3}$}

${ }^{1}$ Principal Investigator, Lyell McEwin Hospital, Australia

${ }^{2}$ Investigator, Lyell McEwin Hospital, Australia

${ }^{3}$ Supervisor, Lyell McEwin Hospital, Australia

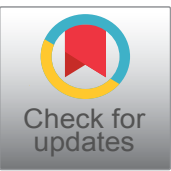

*Corresponding author: Singankutti Mudalige Thanuja Nilushipriyangika, Principal Investigator, Lyell McEwin Hospital, Haydown Road, Elizabeth Vale, SA 5112, Post: 5112, Adelaide, Australia

\begin{abstract}
Background and objective: High flow nasal oxygen (HFNO) has been shown to reduce desaturations during flexible bronchoscopy. We evaluated outcomes of HFNO vs. low flow nasal oxygen (LFNO) on intraprocedural hypoxemia, the demand of sedative and reversal medications, postprocedure complications, procedure conversion to general anaesthesia and patient comfort during outpatient standard and EBUS (Endo Bronchial Ultrasound) guided bronchoscopy under conscious sedation.
\end{abstract}

Methods: A Prospective open-label study where the first 42 patients were allocated to LFNO and subsequent 42 patients were allocated to HFNO. Baseline and lowest oxygen saturation, duration of hypoxemia, procedure type, amount of sedative and reversal medications and patient comfort were assessed.

Results: Both HFNO and LFNO had similar characteristics at baseline. The odds ratio of being hypoxic $(<90 \%)$ was 13.8 times more in the LFNO group ( $\mathrm{Cl} 3.55-70.7, \mathrm{p}<0.001)$ after adjustment for confounders. In addition, the LFNO group had a longer period of desaturation (OR 11.24, Cl $3.18-49.78, p<0.001)$. There was no statistically significant difference in median lowest peripheral oxygen saturation, the amount of sedative and reversal medications, patient comfort, procedure conversion to general anaesthesia, and post-procedure complications between the two groups.

Conclusion: During outpatient standard and EBUS guided bronchoscopy, HFNO is associated with a statistically significant reduction in oxygen desaturation and duration of hypoxemia. However, there was no clinically significant difference in adverse outcomes, or patient comfort between the two groups.

\section{Keywords}

High flow, Low flow, Oxygen, Outpatient bronchoscopy, Conscious sedation

\section{Abbreviations}

HFNO: High Flow Nasal Oxygen; LFNO: Low Flow Nasal Oxygen; TBLB: Transbronchial Lung Biopsy; EBUS: EndoBronchial Ultrasound; TBNA: Trans-Bronchial Needle Aspiration; BAL: Bronchoalveolar Lavage

\section{Introduction}

Bronchoscopy is an invaluable diagnostic tool for many lung disorders and a safe procedure with low morbidity and mortality. Since the introduction of the flexible fibre optic bronchoscope in late 1960 by Shigeto Ikeda, there have been several microtechnological advances in the field of bronchoscopy [1]. Out of these was the introduction of endoscopic ultrasonography which plays an important role in interventional pulmonology [1]. Usually, the procedure is carried out under moderate conscious sedation by using intravenous

Citation: Priyangika SMTN, Alawami M, Karunarathne S (2022) Evaluation of Patient Outcomes of High Flow versus Low Flow Nasal Oxygen during Outpatient Bronchoscopy under Conscious Sedation at Thoracic Procedure Suite in Lyell McEwin Hospital. Int J Respir Pulm Med 9:169. doi.org/10.23937/23783516/1410169

Accepted: February 03, 2022; Published: February 05, 2022

Copyright: (C) 2022 Priyangika SMTN, et al. This is an open-access article distributed under the terms of the Creative Commons Attribution License, which permits unrestricted use, distribution, and reproduction in any medium, provided the original author and source are credited 
sedative medications to facilitate procedural tolerability, diagnostic yield, and safety. Nevertheless, deep sedation which can lead to higher rates of desaturation may be beneficial in complex bronchoscopic procedures to increase the diagnostic yield and safety [2]. Complications during the procedure can be related to the procedure itself or due to anaesthesia [3]. Hypoxemia is one of the commonest procedure-related complications commencing with the administration of sedation and worsening on passage through the vocal cords [4]. In addition, patient positioning, airway suctioning, level of sedation, oxygenation technique, decreasing FEV1, intra-procedural sampling, and definition of oxygen desaturation used may also influence oxygen saturation [2]. The use of preprocedural oxygen and the specific sampling procedure were predictive of a higher rate of desaturation episodes. Bronchoalveolar lavage (BAL) is the commonest sampling procedure that causes hypoxemia followed by wash, brush, and biopsy [4]. There is no demonstrated difference in oxygen saturation with transnasal or trans oral bronchoscopic approaches [5]. The majority of these desaturation events are transient and do not require specific intervention [6]. Supplemental oxygen via nose or mouth can reduce the incidence, degree, or duration of desaturation and should be given to maintain the arterial oxygen saturation at or above $90 \%$ [5]. Supplemental oxygen can be given as high flow (HFNO) or low flow nasal oxygen (LFNO).

HFNO is a therapy that can deliver heated humidified medical gas using an air-oxygen blender at a flow rate of up to $60 \mathrm{~L} / \mathrm{min}$ with an inspiratory oxygen fraction $\left(\mathrm{FiO}_{2}\right)$ ranging from $21 \%$ to $100 \%$ [7]. The main advantages of HFNO are reduction in anatomical dead space, work of breathing, and provision of constant $\mathrm{FiO}_{2}[7,8]$. There is growing evidence to support the use of HFNO in several clinical situations especially in intensive care patients requiring bronchoscopy [9]. However, limited studies in the literature have assessed the benefits of HFNO instead of LFNO during outpatient bronchoscopy including both standard and Endo Bronchial ultrasoundguided (EBUS) bronchoscopy.

We designed this open-label, non-randomized, prospective study to compare the rate of hypoxemia between two groups (primary endpoint) during outpatient bronchoscopy under conscious sedation.

\section{Methods}

All individual, de-identified datasets generated during and or analysed during the study are available from the corresponding author on reasonable request. Ethical approval was given by the Central Adelaide Local Health Network Human Research Ethics Committee, South Australia.

This study compared HFNO with LFNO during outpatient bronchoscopy (standard and EBUS guided) under conscious sedation. It was a prospective nonrandomized study conducted at a single center from April to June 2020. All the procedures were performed in the thoracic procedure suite (TPS) by experienced interventional pulmonologists and a Respiratory fellow with the assistance of well-trained bronchoscopic nursing staff. During the beginning of the Covid 19 pandemic in South Australia, the use of HFNO has been restricted to prevent aerosol transmission. This has led to a change in the practice of HFNO to LFNO during outpatient bronchoscopy by interventional pulmonologists in our unit. During this period with restrictions, we evaluated all the adults (i.e., aged $>18$ years) outpatients undergoing bronchoscopy under conscious sedation with LFNO. When the restrictions were lifted to use HFNO we evaluated the same number of adult patients with HFNO. Eligible criteria were patients: 1) Aged more than 18 years or above; 2) Clinical indication to undergo outpatient bronchoscopy; 3) Capacity to give informed consent. Exclusion criteria were patients: 1) Aged less than 18 years; 2) Contraindication to bronchoscopy; 3) Contraindication to HFNO; 4) Reduced level of consciousness; 5) Pregnancy; 6) Pre-procedure hypoxemia $\left(\mathrm{SpO}_{2}<90 \%\right)$ and patients on long-term oxygen therapy (LTOT).

Patients were screened by one of the study team members and consented to before arrival in the thoracic procedure suite (TPS). 42 patients were selected for each group with a total number of 84 . Baseline characteristics, procedural data including postprocedure complications, and patient comfort were collected. All patients were monitored with non-invasive blood pressure and oxygen saturation. Preprocedural $2 \%$ lignocaine was sprayed into the throat, intravenous Midazolam and Fentanyl were administered. While doing the procedure vocal cords and airways were tropicalized with $2 \%$ lignocaine. Midazolam and Fentanyl are administered depending on demand. Spontaneous respiratory efforts were preserved during the procedure as evidenced by stable hemodynamic variables and depth of sedation was verified by verbal and/or tactile stimuli assessing purposeful response. The first 42 patients were given LFNO with nasal canula inserted into the nostrils starting with 2 litres per min and the flow rate was increased to maintain $\mathrm{SpO}_{2}$ above $90 \%$. Next 42 patients were given HFNO using Optiflow Thrive with a nasal cannula inserted into the nostrils with starting flow rate of 25 litres per min. Like the LFNO group flow rates were increased to maintain $\mathrm{SpO}_{2}$ above $90 \%$. Standard oxygen tubing has been used in both groups.

All bronchoscopies were performed by either interventional pulmonologists or fellow through the oral route utilizing the mouth guard. Oxygen desaturation, duration of desaturation, the dosage of sedative and reversal medications, type of intervention (standard or EBUS and BAL performed or not) were recorded by 
one of the team members while and soon after the procedure.

After the procedure, all the patients were transferred to the recovery area and routine post anaesthetic observations were carried out for 2 hours. Patients with the LFNO group continued to receive oxygen with the low flow if they were hypoxic, in the HFNO group low flow oxygen was given at recovery if they were hypoxic. During the recovery period, all the patients were assessed for post-procedure complications and patient comfort by the principal investigator. Patient comfort was analysed by using four points satisfaction score (very comfortable, comfortable, slightly uncomfortable, uncomfortable) 1 hour after the procedure when the sedation had worn off.

The proportion of patients who desaturated $<90 \%$ at any time during the procedure was the primary outcome. Secondary outcomes included: 1) Duration of hypoxemia; 2) Hypoxemia in different bronchoscopic techniques and sampling; 3) Postprocedure complications; 4) Procedure conversion to general anaesthesia; 5) Amount of sedative and reversal medications usage; 5) Patient comfort between two groups during outpatient bronchoscopy.

\section{Results}

A total of 84 patients were recruited and included in the analysis. 42 patients were included in each group. No patient required supplemental oxygen before sedation and the procedure.

Baseline patient characteristics and procedural data were similar between groups (Table 1).

Regarding the primary endpoint, the proportion of patients desaturating $<90 \%$ was lower in the HFNO group (4 patients, $9.5 \%$ versus 17 patients $(40 \%)$ in the LFNO group $(p=0.001)$. In a logistic regression model with covariates of oxygen delivery device, starting saturation, presence of OSA/COPD, smoking status, procedure type, and $B A L$ being performed, the odds of being hypoxic was 13.8 times higher in LFNO $(\mathrm{Cl} 3.55,70.7, \mathrm{p}<0.001)$. Using the same covariates with the ordered nominal regression model, OR of longer duration of hypoxia was 11.24 (Cl 3.18-49.78, $p<0.001$ ) in LFNO compared to HFNO. However, there was no significant difference in median lowest peripheral oxygen saturation between the two groups. Odds of being more comfortable in the HFNO group were 1.11 times more likely than those in the LFNO group ( $\mathrm{Cl} 0.52-2.5, \mathrm{p}=0.76)$ but there was no statistically significant difference between the two groups.

Table 1: The two groups are similar in terms of baseline characteristics.

\begin{tabular}{|c|c|c|c|}
\hline Characteristic & $\begin{array}{l}\text { high flow, } \\
N=42^{1}\end{array}$ & $\begin{array}{l}\text { low flow, } \\
N=42^{1}\end{array}$ & p-value ${ }^{2}$ \\
\hline Age & $68(59,72)$ & $64(59,72)$ & 0.8 \\
\hline Gender (Number of female (\%)) & $22(52 \%)$ & $14(33 \%)$ & 0.12 \\
\hline Procedure & & & 0.4 \\
\hline Standard bronchoscopy & $14(33 \%)$ & $18(43 \%)$ & \\
\hline Linear EBUS & $19(45 \%)$ & $19(45 \%)$ & \\
\hline Radial EBUS & $9(21 \%)$ & $5(12 \%)$ & \\
\hline Indication & & & 0.7 \\
\hline Chronic cough & $4(9.5 \%)$ & $3(7.1 \%)$ & \\
\hline GGO & $1(2.4 \%)$ & $4(9.5 \%)$ & \\
\hline Haemoptysis & $2(4.8 \%)$ & $3(7.1 \%)$ & \\
\hline Mediastinal/hilar LN & $18(43 \%)$ & $18(43 \%)$ & \\
\hline Pulmonary nodule or Mass & $17(40 \%)$ & $14(33 \%)$ & \\
\hline $\mathrm{BAL}$ & $15(36 \%)$ & $18(43 \%)$ & 0.7 \\
\hline Obesity & $20(48 \%)$ & $13(31 \%)$ & 0.2 \\
\hline $\mathrm{CCF}$ & $2(4.8 \%)$ & $1(2.4 \%)$ & $>0.9$ \\
\hline COPD & $19(45 \%)$ & $19(45 \%)$ & $>0.9$ \\
\hline AF & $5(12 \%)$ & $4(9.5 \%)$ & $>0.9$ \\
\hline OSA & $8(19 \%)$ & $7(17 \%)$ & $>0.9$ \\
\hline Smoking status & & & $>0.9$ \\
\hline Never smoked & $7(17 \%)$ & $8(19 \%)$ & \\
\hline Active smoker & $13(31 \%)$ & $13(31 \%)$ & \\
\hline Ex-smoker & $22(52 \%)$ & $21(50 \%)$ & \\
\hline \multicolumn{4}{|l|}{${ }^{1}$ Median (IQR); n (\%) } \\
\hline${ }^{2}$ Wilcoxon rank sum test; Pears & & & \\
\hline
\end{tabular}


Table 2: Outcomes: The table shows both groups are similar in terms of procedural outcomes apart from oxygen flow (intervention) and lowest oxygen saturations measured during bronchoscopic procedures. This is not surprising as we expected that patients on lower flow of oxygen will have lower saturations during procedure. However, this didn't result in any more complications compared with the other group.

\begin{tabular}{|c|c|c|c|}
\hline Characteristic & $\begin{array}{l}\text { high flow, } \\
\mathrm{N}=42^{1}\end{array}$ & $\begin{array}{l}\text { low flow, } \\
\mathrm{N}=\mathbf{4 2}^{1}\end{array}$ & p-value ${ }^{2}$ \\
\hline Fentanyl dose (mg) & $100(75,125)$ & $100(75,125)$ & 0.6 \\
\hline Midazolam dose (mg) & $5.00(4.00,6.00)$ & $5.00(4.00,6.00)$ & 0.2 \\
\hline Need to reverse sedation & $2(4.8 \%)$ & $1(2.4 \%)$ & $>0.9$ \\
\hline Flow rate at the start of the case (L/min) & & & $<0.001$ \\
\hline 2 & $0(0 \%)$ & $42(100 \%)$ & \\
\hline 25 & $42(100 \%)$ & $0(0 \%)$ & \\
\hline Max flow rate (L/min) & $25(25,25)$ & $4(2,8)$ & $<0.001$ \\
\hline Starting peripheral saturation (\%) & & & 0.6 \\
\hline $95-100 \%$ & $41(98 \%)$ & $39(93 \%)$ & \\
\hline $90-94 \%$ & $1(2.4 \%)$ & $3(7.1 \%)$ & \\
\hline Lowest peripheral saturation (\%) (intraprocedure) & $97.0(96.0,97.0)$ & $92.0(88.0,95.0)$ & $<0.001$ \\
\hline Duration of desaturations (min) & & & 0.007 \\
\hline 0 & $38(90 \%)$ & $25(60 \%)$ & \\
\hline 3 & $0(0 \%)$ & $1(2.4 \%)$ & \\
\hline 5 & $0(0 \%)$ & $5(12 \%)$ & \\
\hline 10 & $2(4.8 \%)$ & $3(7.1 \%)$ & \\
\hline 15 & $2(4.8 \%)$ & $3(7.1 \%)$ & \\
\hline 30 & $0(0 \%)$ & $4(9.5 \%)$ & \\
\hline 120 & $0(0 \%)$ & $1(2.4 \%)$ & \\
\hline Post procedure hypoxia $\left(\mathrm{SpO}_{2}<90 \%\right)$ & $0(0 \%)$ & $1(2.4 \%)$ & $>0.9$ \\
\hline Bronchospasms & $0(0 \%)$ & $1(2.4 \%)$ & $>0.9$ \\
\hline Hypotension & $2(4.8 \%)$ & $0(0 \%)$ & 0.5 \\
\hline MERT calls & $0(0 \%)$ & $0(0 \%)$ & \\
\hline Pneumothorax & $0(0 \%)$ & $0(0 \%)$ & \\
\hline Respiratory failure & $0(0 \%)$ & $0(0 \%)$ & \\
\hline Patient comfort & & & 0.3 \\
\hline Very comfortable & $12(29 \%)$ & $8(19 \%)$ & \\
\hline Comfortable & $13(31 \%)$ & $20(48 \%)$ & \\
\hline Slightly uncomfortable & $11(26 \%)$ & $6(14 \%)$ & \\
\hline Very uncomfortable & $6(14 \%)$ & $8(19 \%)$ & \\
\hline Sedation converted into general anaesthesia & $0(0 \%)$ & $2(4.8 \%)$ & 0.5 \\
\hline Type 2 respiratory failure (Hypercapnic) & $1(2.4 \%)$ & $0(0 \%)$ & $>0.9$ \\
\hline Unexpected admission to ward & $1(2.4 \%)$ & $0(0 \%)$ & $>0.9$ \\
\hline \multicolumn{4}{|l|}{${ }^{1}$ Median (IQR); n (\%) } \\
\hline
\end{tabular}

In terms of secondary endpoints, there was no significant difference between groups in terms of sedative medications used, need to use reversal, postprocedure complications, and unplanned admission to the ward (Table 2).

Pearson's Chi-squared test was used for categorical data where the frequency was more than 5 in each category while Fisher's exact test was used when the frequency was 5 or less. Continuous data were presented as a median with interquartile range and the
Wilcoxon rank-sum test was performed. Analysis was carried out using $\mathrm{R}$ (version 4.0.2).

\section{Discussion}

This single-institution study in a tertiary care hospital confirms that outpatient bronchoscopy performed with HFNO under conscious sedation is associated with a statistically significant reduction in intraprocedural oxygen desaturation and duration of hypoxemia compared to LFNO. However, there is no difference in median lowest peripheral oxygen saturation, immediate 
post-procedure complications, patient comfort, the demand of sedative and reversal medications usage, procedure conversion to general anaesthesia in the two groups. As there was no statistically significant difference in secondary outcomes including safety, we would like to highlight that LFNO can also be considered safe to be used during outpatient bronchoscopy in less complex patients and is cost-effective. However, further studies are needed.

Hypoxemia is a common complication during fiberoptic bronchoscopy and $\mathrm{PaO}_{2}$ can be dropped up to $20 \mathrm{mmHg}$ [10]. Rokach, et al. showed that octogenarians are more prone to develop significant hypoxemia during bronchoscopy [11]. CHEST Quality Improvement Registry, Evaluation, and Education (AQuIRE) reported a $0.3 \%$ complication rate of sustained hypoxemia during EBUS-TBNA, and factors associated with escalation of care were old age ( $>70$ years), inpatients status, deep sedation, or general anaesthesia [12].

Up to now, there are only four published studies compared the outcome of high flow and low flow nasal oxygen during bronchoscopy. To our knowledge, this is the first study that compared standard and EBUS bronchoscopy together under moderate conscious sedation.

Douglas, et al. analysed patients undergoing EBUS bronchoscopy but did not find statistical significance in desaturations between two groups [13]. However, this reached significance when analysed on a per-protocol basis, but this was following a movement of a single patient between groups [2,13]. Secondary outcomes of this study found that median lowest $\mathrm{SpO}_{2}$ during the procedure and preoxygenation $\mathrm{SpO}_{2}$ were significantly higher in the HFNO group but no difference in other secondary outcomes including safety and patient satisfaction.

Ben-Menachem, et al. demonstrated that HFNO significantly reduces intraprocedural desaturations, number of airway interventions, and procedure interruptions in post-transplant lung population undergoing transbronchial lung biopsies (TBLB) [2]. Compared to other studies this study was specifically undertaken in lung transplant recipients booked for TBLB who were more prone to desaturate due to impaired respiratory reserve, required lengthy procedure, and deep level of sedation. Nevertheless, patient satisfaction and post-procedure complications were similar in both groups.

Longini, et al. evaluated outpatients who had standard bronchoscopy and BAL and like the previous two studies found that HFNO provides better oxygenation than LFNO during BAL with a similar safety profile in both groups [8].

Irfan, et al. evaluated a group of patients who underwent EBUS TBNA under conscious sedation and showed that HFNO is associated with significantly better oxygenation compared with LFNO with a similar safety profile, patient comfort, and their willingness to return for a repeat procedure [14]. They emphasized that HFNO can potentially allow deeper sedation and better yield while reducing the incidence of repeat procedures and would be cost-effective in centers where EBUSTBNA is performed under general anaesthesia.

Generally, our results are also consistent with other studies.

What differs in our study is that we have evaluated outpatients who had either standard or EBUS bronchoscopic procedures under conscious sedation. Previously published studies were mainly done on specific bronchoscopic procedures and sampling namely EBUS TBNA, Post lung transplant TBLB, and BAL. We agree with previous authors that HFNO can provide better oxygenation during the procedure with a minimum duration of hypoxemia. But on the other hand, both groups have similar safety profiles, patient comfort, median lowest peripheral oxygen saturation, demand for sedative and reversal medications, procedure conversion to general anaesthesia.

Bronchoscopic procedures, including preparation and recovery time, typically take about four hours. However, the bronchoscopic procedure itself typically lasts for 15 to 60 minutes [15]. Therefore, the oxygen requirement for a single procedure using LFNO will be $60 \mathrm{~L}$ to $240 \mathrm{~L}$ (assuming the median oxygen flow rate is $4 \mathrm{~L}$ per minute). For the same procedure using HFNO, this will be $375 \mathrm{~L}$ to $1500 \mathrm{~L}$ (assuming the minimum oxygen flow rate is $25 \mathrm{~L}$ per minute). The oxygen requirement for a bronchoscopic procedure using HFNO is more than six times compared to LFNO.

This points up the extra oxygen required for a bronchoscopic procedure using HFNO. In addition, HFNO is not widespread currently due to the limited access to the required equipment, especially in resources poor settings.

All the studies including ours have demonstrated that both HFNO and LFNO are safe during bronchoscopy under conscious sedation, but LFNO can significantly reduce the cost and oxygen usage. Furthermore, HFNO is associated with an increased risk of aerosol transmission of infective pathogens specially Covid-19.

Our study was a real-world evaluation of HFNO vs. LFNO during outpatient bronchoscopy under conscious sedation. Compared to outpatients, inpatients requiring bronchoscopy are more clinically unstable and we haven't evaluated their outcome from our study.

\section{Conclusion}

Compared to low flow nasal oxygen (LFNO), high flow nasal oxygen (HFNO) is associated with a statistically significant reduction in intraprocedural oxygen 
desaturation and duration of hypoxemia. However, both groups demonstrated a similar safety profile, median lowest peripheral oxygen saturation, and patient comfort. LFNO is associated with a low cost due to lower oxygen requirements and equipment costs.

Wewould like to highlight that LFNOcan be considered safe to be used during outpatient bronchoscopy under conscious sedation in less complex patients. However, further multicentre prospective studies are needed with a particular emphasis on identifying high-risk patients who would benefit most from HFNO.

\section{Limitation}

This study could have enrolled more cases to increase the power of the study. Due to the Covid-19 restriction randomisation could not be performed.

\section{Acknowledgements}

The authors would like to acknowledge the nursing staff in Lyell McEwin Hospital, South Australia for their support given in thoracic procedure suites during the procedure.

\section{Disclosure Statement}

The authors declare that they have no competing interests.

\section{Authors' Contribution}

Singankitti Mudalige Thanuja Nilushi Priyangikaconceptualization, investigation, project administration, methodology, data curation, writing original draft; Moayed Alawami-formal analysis and software; Shanka Karunarathne-conceptualization, project administration, resources, supervision.

\section{References}

1. Dooms C, Seijo L, Gasparini S, Trisolini R, Ninane V, et al. (2010) Diagnostic bronchoscopy: State of the art. Eur Respir Rev 19: 229-236.

2. Ben-Menachem E, McKenzie J, O'Sullivan C, Havryk AP (2020) High-flow nasal oxygen versus standard oxygen during flexible bronchoscopy in lung transplant patients. J Bronchology Interv Pulmonol 27: 259-265.
3. Lukomsky GZ, Ovchinnikov AA, Bilal A (1981)Complications of bronchoscopy: Comparison of rigid bronchoscopy under general anesthesia and flexible fiberoptic bronchoscopy under topical anesthesia. Chest 79: 316-321.

4. Chhajed PN, Glanville AR (2003) Management of hypoxemia during flexible bronchoscopy. Clin Chest Med 24: $511-516$

5. Du Rand IA, Blaikley J, Booton R, Chaudhuri N, Gupta V, et al. (2013) British Thoracic Society guideline for diagnostic flexible bronchoscopy in adults: Accredited by NICE. Thorax 68: 1-44.

6. Jones AM, O'Driscoll R (2001) Do all patients require supplemental oxygen during flexible bronchoscopy? Chest 119: 1906-1909.

7. Sharma S, Danckers M, Sanghavi D, Chakraborty RK (2021) High flow nasal cannula. In: Stat Pearls. Treasure Island (FL): Stat Pearls Publishing.

8. Longhini F, Pelaia C, Garofalo E, Bruni A, Placida R, et al. (2022) High-flow nasal cannula oxygen therapy for outpatients undergoing flexible bronchoscopy: A randomised controlled trial. Thorax 77: 58-64.

9. Drake MG (2018) High-flow nasal cannula oxygen in adults: An evidence-based assessment. AATS 15: 145-1555.

10. Albertini RE, Harrell JH, Kurihara N, Moser KM (1974) Arterial hypoxemia induced by fiberoptic bronchoscopy. JAMA 230: 1666-1667.

11. Rokach A, Fridlender ZG, Arish N, Berkman N (2008) Bronchoscopy in octogenarians. Age and Ageing 37: 710713.

12. Eapen GA, Shah AM, Lei $X$, Jimenez CA, Morice RC, et al. (2013) Complications, consequences, and practice patterns of endobronchial ultrasound-guided transbronchial needle aspiration. Chest 143: 1044-1053.

13. Douglas $\mathrm{N}, \mathrm{Ng}$ I, Nazeem F, Lee $\mathrm{K}$, Mezzavia $\mathrm{P}$, et al. (2018) A randomised controlled trial comparing high-flow nasal oxygen with standard management for conscious sedation during bronchoscopy. Anaesthesia 73: 169-176.

14. Irfan M, Ahmed M, Breen D (2021) Assessment of high flow nasal cannula oxygenation in endobronchial ultrasound bronchoscopy: A randomized controlled trial. J Bronchology Interv Pulmonol 28: 130-137.

15. American Thoracic Society (2015) Flexible bronchoscopy. Am J Respir Crit Care Med 191: 7-8. 